\title{
Derechos de los animales, deberes de los humanos
}

\author{
M. ${ }^{\mathrm{a}}$ TERESA LÓPEZ DE LA VIEJA \\ Universidad de Salamanca
}

RESUMEN. La Ética aplicada se suele referir a los problemas prácticos e intereses de los seres humanos. Sin embargo, las cuestiones éticas se han ido ampliando en los últimos años. A pesar de que el punto de vista moral sigue anclado en un enfoque antropocéntrico, el medio ambiente y los seres vivos son ahora objeto de alguna consideración moral. La justicia, como principio moral, puede ampliarse también hasta incluir a los no humanos, sus intereses y su bienestar. Desde un enfoque antropocéntrico moderado, el artículo defiende en este tema una consideración moral asimétrica; pues sólo podemos entender los derechos de los animales como resultado de los deberes de los humanos hacia la propia especie. Son deberes directos hacia las generaciones futuras de humanos, deberes indirectos hacia los no humanos. Por tanto, la justicia entre especies amplía la idea de justicia entre generaciones.

Los humanos han causado y causan daños en el medio ambiente, amenazan seriamente la continuidad de las demás especies, se comportan a menudo de forma innecesariamente cruel hacia los animales.
Abstract. Applied Ethics usually refers to human beings, to their practical problems and to their interests. However, the sphere of ethical issues has expanded during the last years. In spite of the fact that the moral point of view is still grounded on anthropocentrism, the environment and living beings are now the aim object of some moral concern. Justice, as a moral principle, could expand itself, as well, in order to include nonhuman beings: their interest and their welfare. From a moderate anthropocentric standpoint, this article defends an asymmetrical moral concern. Indeed, we could understand animal's rights only as a result of human duties towards the own species. This essay claims for direct duties to future generations of humans, and for indirect duties to nonhuman. Therefore, justice between species would enlarge justice between generations.

Ahora bien, ¿cómo propiciar el respeto más allá de la propia especie? ¿El interés genuino por los no humanos? La información existente sobre crueldad, falta de interés, abusos, demuestran la resistencia a 
aceptar el valor intrínseco de los animales. Estando así las cosas, el valor de los no humanos parece todavía un argumento débil a la hora de modificar la actitud hacia otras especies. ¿Hay argumentos más consistentes para defender conductas sensibles hacia el bienestar animal? El artículo justifica un trato más civilizado hacia los animales no humanos y la preservación de las especies, desde un enfoque antropocéntrico moderado. $\mathrm{Y}$ desde una Ética medioambiental. Esto es, sin necesidad de renunciar a la primacía - que no privilegio- de la especie humana, asumiendo a todos los efectos que los recursos y las especies no humanas forman parte del «medio ambiente». En cuanto a la tesis de los deberes indirectos - de los humanos hacia los no humanos-, ésta se apoya en la existencia de obligaciones directas hacia los humanos. Sólo que del interés hacia las generaciones futuras se derivan también ciertas obligaciones hacia los animales. Pues el bienestar de los humanos - en el presente y en el futuro- está ligado a la supervivencia de las demás especies.

¿Un cálculo interesado? En realidad, se trata de limitar la conducta de los seres humanos, en la medida que ésta supone un riesgo para la continuidad de otras especies. Se podría apelar también a un argumento resbaladizo: paso a paso, la violencia hacia los animales nos hace insensibles al sufrimiento. Y luego podríamos aceptar cualquier otra forma de violencia. La crueldad degrada también a quienes la practican. Argumentos de este tipo indican que un enfoque antropocéntrico puede ser tanto o más eficaz que la apelación a los intereses o al valor intrínseco de los animales. La defensa del medio ambiente y de los no humanos surge, por así decirlo, de un doble compromiso, hacia la propia especie y hacia otros seres vivos. Por ejemplo, los «deberes indirectos» comprometen de alguna manera a los agentes, al margen de cual sea su opinión sobre el valor intrínseco de los no humanos, su capacidad para percibir o para tolerar el sufrimiento ajeno, su interés por el medio ambiente. A favor de la estrategia antropocéntrica -antropocéntrica moderadajuega también el hecho de que las políticas ambientales han de ser debatidos en la esfera pública y en relación al bienestar de los ciudadanos. Valga como muestra de esto ultimo la posición de A. Roy ${ }^{1}$. Esta escritora ha rechazado durante años la construcción de grandes presas en la India. Porque la intervención sobre el medio ambiente debe servir para la igualdad, no para crear pobreza. No hay, en su opinión, razones sólidas para que 40 o 50 millones de personas hayan de ser desplazadas por la construcción de las presas en el Valle de Narmada.

Cada vez tiene más defensores la tesis de que la protección de las especies y de los recursos ambientales está vinculada a un cambio radical de la sensibilidad moral. Cambio que requiere también voluntad política. Por muchas razones, lo verde ${ }^{2}$ es, también, rojo. Dicho en breve, las relaciones de «justicia entre especies» derivan de alguna forma de «justicia entre generaciones». Este punto de vista se mantendrá a lo largo del artículo, en la línea del antropocentrismo moderado ${ }^{3}$ : 1) Los animales merecen alguna consideración, en tanto destinatarios o «pacientes» de la intervención de los humanos sobre el medio ambiente. 2) Puesto que la continuidad entre las especies puede ser entendida en términos biocéntricos o en términos antropocéntricos, dependiendo de cuál sea el enfoque, se hablará de «derechos» o, por el contrario, de «deberes» de los humanos hacia los no humanos. Por lo tanto, la consideración moral de los no humanos sólo generará deberes indirectos de los humanos hacia los no humanos. 3) Las conductas destinadas a evitar el daño -entre humanos - y las conductas destinadas a la preservación de las especies- podrán ser justificadas 
por analogía, desde una teoría de la justicia. Esto es, la preservación de los animales no humanos genera obligaciones. Deberes de los humanos.

\section{LOS ANIMALES COMO «PACIENTES» MORALES}

«Lo personal es político...» ${ }^{4}$, para el Feminismo esto significa que se debe cuestionar a fondo la separación estricta entre esfera pública y esfera privada, con objeto de terminar con aquellas formas de discriminación que toman el género como pretexto. Política de igual dignidad $y$, ante todo, derechos especiales para grupos minoritarios, según los defensores del Multiculturalismo ${ }^{5}$. En el punto de mira de casi todos los movimientos sociales se encuentran aquellos prejuicios sobre los que se construyó un mundo escindido, injusto. Los derechos especiales y la legislación en apoyo de la igualdad son, pues, el resultado de la presión de los movimientos sociales y, en otro nivel, el resultado de una amplia transformación de lo político. Las formas de esclavitud y de opresión deben retroceder, sin duda, ante la nueva expansión de los derechos. Derechos como «triunfos» ${ }^{6}$. Sin embargo, el final de la antigua esclavitud estaría al alcance tan sólo de aquellos que estuvieran en situación de hacerse oír, aunque se expresasen todavía con dificultad, o con «otra voz» ${ }^{7}$. Al parecer, la emancipación tiene aún un límite rígido: desde y para la especie humana. Y esta frontera dificulta la extensión de los medios para la protección de especies, así como los derechos ${ }^{8}$ de los no humanos.

¿Existen motivos reales para conservar a ultranza esa frontera? ${ }^{9}$. Desde los años setenta, los movimientos en favor de la liberación de los animales vienen alegando que el proceso de civilización ha de seguir hacia adelante, a fin de incluir a los animales no humanos y al medio ambiente en el ámbito moral. P. Singer ${ }^{10}$ considera que la liberación de los animales es importante para la especie humana, para la expansión de su horizonte moral. Y, sólo por eso, esta cuestión tendría que ser recogida por la Teoría. La moralidad ha estado asociada siempre a los humanos, en tanto seres con autonomía, con lenguaje y con autoconciencia; sin embargo, ralguna de estas características se encuentran también en los no humanos, si bien en grado distinto. De forma análoga, podemos atribuirles derechos, aunque no los mismos derechos, según precisa este autor. Tampoco cree que hayan estado justificados los criterios utilizados para comparar el valor de los seres vivos ${ }^{11}$. ¿Qué razones hay para atribuir menor valor a individuos con similar capacidad a la de un ser humano, sólo por el hecho de no pertenecer a la especie humana? ¿Por qué son tan distintas nuestras reacciones ante la muerte de un ser humano y la muerte de un chimpancé, por ejemplo? Si rechazamos prejuicios como el racismo, ¿por qué mantenemos todavía el «especieísmo» o prejuicio de especie? En realidad, el sufrimiento es igual para todos, importa poco la especie a la cual pertenezca un ser vivo. Así pues, la definición de los no humanos como seres inferiores ha sido y aún es el principal impedimento para llegar a un enfoque completamente libre de prejuicios: la igual consideración de intereses. Los trasplantes con órganos de animales o xenotrasplantes ejemplifican para $P$. Singer la vigencia de tales prejuicios, el especieísmo y la consideración desigual de los seres vivos.

En un sentido similar - a pesar de algunas diferencias significativas entre ambos-, T. Regan ${ }^{12}$ rechaza el uso de los animales como recursos a disposición de los humanos. Para ello, defiende una versión de continuidad entre especies, con objeto de justificar la igual consideración de todos los intereses. Este autor insiste en que el status moral ${ }^{13}$ de los seres vivos no ha de estar ligado al estado de las capacidades mentales, por eso defiende que los 
animales tienen derechos. El derecho a no sufrir daño, exactamente como en el caso de los humanos. Sin embargo, este «derecho» no es susceptible de prueba. No de la misma manera que atribuimos derechos a los humanos. Los derechos aplicados a los animales pretenden limitar ciertas conductas, terminando con prácticas industriales que provocan sufrimiento $u$ otros daños importantes. Esto sería posible si el principio de la vida como algo valioso se extendiera a los no humanos. Por lo tanto, un genuino interés por el bienestar de los animales que comparten el entorno constituiría un paso fundamental, a fin de llegar al reconocimiento de sus derechos morales. En cambio, otros autores prefieren hablar de «derechos» ${ }^{14}$ en el sentido de medidas para la preservación de los animales y para la consideración de su bienestar. La mayoría de las aportaciones insisten en que se debe producir un cambio radical en el trato hacia los animales, demostrando que la Filosofía contemporánea toma cada vez más en serio esta cuestión.

Algo bien distinto es que haya consenso en torno a los modelos teóricos ${ }^{15}$. $\mathrm{El}$ enfoque es importante, ya que no se trata de promover actitudes compasivas o el afecto hacia los seres vivos ${ }^{16}$, sino de algo más radical. Se trata de cambiar los principios morales. Principios que sean compatibles con el reconocimiento de derechos a otras especies, haciendo frente así a una larga tradición de antropocentrismo radical. Entre tanto, los nuevos derechos chocan con creencias bien asentadas en la cultura occidental. Por ejemplo, la convicción de que existe una clara jerarquía entre los seres vivos. $O$ el temor de que la expansión de los derechos reste legitimidad de aquellas prácticas contrarias al bienestar de los no humanos. En suma, cualquier avance en los «derechos» de otras especies pone en tela de juicio los exagerados privilegios, que han sido atribuidos a la especie humana, y la comprensión distorsionada ${ }^{17}$ de los seres vivos, legado de la cultura moderna. Con los datos hoy disponibles, ¿cómo ignorar que algunos no humanos tienen cierto nivel de conciencia? ${ }^{18}$

A un nivel distinto, este tipo de derechos incide en el lenguaje filosófico; por ejemplo, en el uso del concepto de igualdad, cuando nos referimos a la «igual consideración» de intereses en los humanos y en los no humanos. Por estos y otros motivos similares, se hablará de protección ${ }^{19} \mathrm{o}$ de «derechos de los animales», como un triunfo $y$, a la vez, como de un reto importante para el homo mensura rerum. Ahora bien, ¿por qué resulta tan complicado defender la protección de especies y la desaparición de la crueldad? Para empezar, este tipo de argumentos introduce principios relativamente insólitos en la Filosofía moderna, y poco frecuentes aún en la Filosofía contemporánea. Y, sobre todo, los animales no humanos y los humanos están en una situación asimétrica, de ahí la dificultad para extender el ámbito de la moralidad y los derechos.

\section{BIOCENTRISMO Y ANTROPOCENTRISMO}

$P$. Singer ${ }^{20}$ repetía que cuestionar el prejuicio de especie, o tomar en cuenta los intereses de los no humanos, no es lo mismo que hablar de la igualdad de la mujer. Un ejemplo no muy afortunado, sin embargo. En todo caso, considerar los intereses de otras especies quiere decir protegerlas, evitar su sufrimiento, no quiere decir atribuirles derecho al voto, por ejemplo. Por lo tanto, se trata de «derechos», pero no en el sentido en que hablamos de los derechos humanos. El objetivo es extender tales derechos, pues éstos ayudan a institucionalizar la consideración moral hacia los animales, tal como ha defendido B. E. Ro1 lin ${ }^{21}$. Si el derecho a vivir, que corresponde o se atribuye primero a los humanos, no se basa realmente en una característica 
especial - como ser racional o tener conciencia, que eran los argumentos de I. Kant-, sino en tener una vida -no sólo en estar vivo, en sentido biológico- entonces, los animales deben tener también derechos. Pues tienen vida, una vida propia. Defiende esta tesis J. Rachels ${ }^{22}$, quien, a renglón seguido, precisa que los seres vivos tienen diferente valor. En cambio, otros autores como R. G. Frey ${ }^{23}$ se muestran en desacuerdo con este tipo de argumentos relativos al trato hacia los animales en términos de derechos. Cabe entonces hacerse la pregunta ¿qué significa exactamente la «igual consideración de intereses»?

En otro contexto teórico, R. Dworkin ha precisado que no basta la igualdad simple, entendida como una misma distribución de los bienes y de las oportunidades. En realidad, «igual consideración y respeto» ${ }^{24}$ significa derecho a diferentes libertades. Para evitar una versión simplificada de esta noción, P. Westen ${ }^{25}$ ha insistido también sobre la ambivalencia de la igualdad, un término comparativo en todo caso. En tanto que promover la igualdad significa también hacer algunas comparaciones, ¿cómo evitar el antropocentrismo? La expansión de la moralidad invita a respetar a los no humanos «como si» fueran nuestros iguales. Si éste va a ser el principal argumento, entonces la igualdad -como quiera que se entienda - parece que llevará de nuevo al antiguo prejuicio de especie. Veremos ahora que la Ética ha desarrollado ambos lenguajes, antropocéntrico y biocéntrico, a fin de justificar la nueva consideración moral del medio ambiente y de los seres vivos.

1) Nadie se atrevería hoy a defender -al menos no se atrevería en público-, comportamientos crueles hacia los animales no humanos. Así, por ejemplo, buena parte de los experimentos rutinarios con animales carecen hoy en día de justificación ${ }^{26}$. En la mayoría de las granjas ${ }^{27}$ apenas si se tienen en cuenta las necesidades de los animales. $Y$ en países industrializa- dos no existen motivos razonables para practicar la caza ${ }^{28}$, ya que, a diferencia de los métodos tradicionales, sus nuevas formas tienen un gran impacto ambiental y amenazan la preservación de especies. En fin, nadie puede ignorar a estas alturas que la intervención de los humanos modifica el entorno, e influye sobre la disponibilidad de los recursos. La Ética medioambiental ha asumido esta nueva sensibilidad, reconociendo que los no humanos sí tienen algún tipo de status moral ${ }^{29} \mathrm{y}$, por tanto, los consabidos prejuicios de especie ${ }^{30}$ carecen realmente de justificación. De forma muy clara, P. Singer ha venido refiriéndose desde hace años a la «expansión de círculo moral» ${ }^{31}$. Esto quiere decir interesarse por el bienestar de otras especies, promover actitudes altruistas hacia los seres, todos los seres, que sean capaces de sentir dolor y placer. Según esto, no habría motivo de peso para que las demás especies no fueran también beneficiarias de la consideración moral. Entonces ¿qué justifica la diferencia entre el valor moral de humanos y el de los no humanos? De forma similar, J. M. Buchanan ${ }^{32}$ ha explicado que es posible valorar a los animales no humanos como miembros de la propia comunidad. De hecho, algunos animales forman parte del entorno o de la cadena vital en que se desenvuelve la especie humana. Al mismo tiempo, este autor sostiene que los animales no humanos no deben ocupar el mismo lugar que los humanos en el orden moral. Esto es, habrá que distinguir entre «comunidad moral» y «orden moral». A pesar del refinamiento conceptual de esta clase de enfoques, la nueva sensibilidad moral -o expansión de la comunidad moral- ha interesado a otras teorías, de diferente orientación. La Ética ha adoptado, de hecho, varios enfoques para defender objetivos tan razonables como el final de la irresponsabilidad de los humanos, la desaparición de comportamientos crueles hacia otras especies, restricciones para la experi- 
mentación con seres vivos, etc. Y una suerte de emancipación para los no humanos, en sentido análogo a como nos hemos venido refiriendo a otras modalidades de emancipación. Así pues, de una parte, están los argumentos centrados todavía -aunque de manera no excluyente- en la especie humana $y$, de otra, aquellos otros que están centrados en los no humanos: biocentrismo y antropocentrismo.

2) El biocentrismo ${ }^{33}$ aboga por el respeto incondicional hacia el medio ambiente y hacia los seres vivos. Por haber asumido el «igualitarismo biológico» ${ }^{34}$, como lo denominó en su momento A. Naess, Por su parte, M. W. Fox ${ }^{35}$ ha usado los términos «conciencia ecocéntrica» y «Moral ecoética» para defender el valor intrínseco de toda forma de vida y la expansión de los derechos, con objeto de atender en modo adecuado a las necesidades e intereses de los animales. Cualquiera que sea la fórmula elegida por quienes están comprometidos con tal postura, la consideración moral ${ }^{36}$ por la cual abogan se seguirá del principio de que los seres vivos han de ser considerados como intrínsecamente valiosos. No sólo como medios al servicio de la especie dominante, al modo tradicional. Reconocer el valor de los no humanos, su diversidad, significa, entre otras cosas, cambiar o, al menos cuestionar, la prioridad de la supervivencia y del bienestar humanos. Implica modificar drásticamente el comportamiento de la propia especie ${ }^{37}$. En consecuencia, el «uso» ${ }^{38}$ de los animales debería evolucionar decididamente hacia «trato», respeto hacia los animales. No son evidentes, sin embargo, los criterios que permitirán incluir ${ }^{39}$ o excluir a las diferentes especies de la nueva consideración moral. ¿Será la capacidad de sentir dolor? ¿Bastaría con pertenecer a la clase de los seres vivos? ¿Seres con capacidad para tener intereses? La discusión ha avanzado en este terreno, aunque siguiendo vías paralelas. La Ética centrada en los seres vivos, sin restriccio- nes, argumenta a favor de un giro radical, como único medio para llegar a la consideración moral plena. El biocentrismo radical enfatiza el valor intrínseco de los seres vivos y reconoce la existencia de una «comunidad biótica» ${ }^{40}$ cuyo equilibrio no debe ser alterado. En cambio, el biocentrismo moderado reconoce que no todo organismo es esencial ${ }^{41}$ para ese equilibrio y que, sin duda, importa la supervivencia de la especie humana.

3) El antropocentrismo radical apenas si modifica el esquema clásico ${ }^{42}$, en el cual la frontera entre especies otorgaba de manera casi automática primacía a los intereses de la propia especie. En consecuencia, los principios serán independientes - como ya lo fueron antes- de las nuevas realidades ambientales. Dejando atrás una visión rudimentaria sobre la conducta de los animales no humanos - como la visión de R. Descartes-, el antropocentrismo recomienda hoy actitudes más civilizadas. Con todo, no distingue aún claramente entre valores centrados en los humanos y, de otra parte, valores creados ${ }^{43}$ por los humanos aunque de alcance más amplio. No obstante lo cual, los riesgos ambientales han llegado a ser hoy lo suficientemente graves - «comunidad de riesgos compartidos» ${ }^{44}$ es la expresión usada por J. Habermas - como para examinar de nuevo estos principios desde otra óptica, la optica medioambiental. En cambio, el antropocentrismo moderado expande «hacia adelante» - J. Rawls ${ }^{45}$ se refiere al principio de justicia- el radio de influencia de la consideración moral. Por un lado, mantiene la primacía de la especie - tal como sostiene M. Warnock ${ }^{46}$-, por otro, rechaza la crueldad hacia otras especies. En cualquier caso, la moralidad pertenece a los humanos, dado que se desarrolla en el marco de sus relaciones y sus prácticas. Las prioridades humanas estarían justificadas en tanto sirvan a la conservación o a la defensa de la propia especie ${ }^{47}$. La novedad estriba en que ninguna forma de 
antropocentrismo justifica la crueldad o el daño innecesario hacia otras especies. ¿Cómo armonizar las diferencias con lo que tienen en común las especies? A modo de respuesta, E. Tugendhat ${ }^{48}$ justifica la integración de los animales, como si fueran un «anexo» de la comunidad moral. $\mathrm{Al}$ no haber asumido el valor intrínseco de los no humanos ${ }^{49}$, al no aceptar la versión simple de la «igual consideración de intereses», el antropocentrismo menos radical propone, en fin, una consideración moral diferenciada, asimétrica. Esto es, entiende que los no humanos no son en realidad «agentes», sino «pacientes» ${ }^{50}$ morales. Esta segunda versión presenta a los animales no humanos como destinatarios de la acción, a los humanos como responsables últimos de las consecuencias que puedan tener luego sus actos sobre el medio $y$ sobre los seres vivos.

Aun así, la consideración de los animales no humanos como epacientes morales» no asegura que sus intereses sean atendidos en igualdad de condiciones. Está claro que el paso dado introduce mecanismos para establecer ciertas analogías y, lo más interesante, para trabajar en favor de un punto de vista moral ampliado. Pero este nuevo enfoque no garantiza la «liberación» de los animales. Visto el tema en su conjunto, la nueva sensibilidad ambiental llegaba a través de dos enfoques: el «biocéntrico»y el «antropocéntrico». En el primero, lo correcto era hablar de «derechos» de los animales. En cambio, las versiones antropocéntricas se inclinaban casi siempre por los «deberes» hacia los animales o, en todo caso, por un uso derivado o limitado de los «derechos» de los animales no humanos. Porque tales derechos se refieren más bien a compromisos y a obligaciones de la especie humana hacia el medio ambiente, hacia otras especies, y hacia la propia especie ${ }^{51}$. Por tanto, la conciencia ambiental será compatible con prácticas que no generen un daño ${ }^{52}$ innecesario a los animales no humanos. Lo cual no significa que haya que modificar en modo drástico las prioridades: los humanos son lo primero. Significa, en cambio, rechazar la crueldad, asumiendo al mismo tiempo el daño que pueda generar la satisfacción de las necesidades humanas. ¿Qué experimentos con animales son estrictamente necesarios? ¿Por qué tolerar por más tiempo aquellas prácticas que causan sufrimientos inútiles?

Digamos que de la nueva sensibilidad ambiental no se sigue la obligación de seguir, por ejemplo, una dieta vegetariana ${ }^{53}$. Se sigue, en cambio, el compromiso y la responsabilidad hacia el entorno y hacia las especies no humanas. De forma análoga - aunque no idéntica- a como entendemos los compromisos y las responsabilidades hacia los humanos. En conclusión, la asimetría no parece ser un impedimento, sino la principal razón para reclamar con insistencia una nueva consideración moral, así como mayor respeto por los intereses y por el bienestar de los seres vivos. Sobre todo para los seres que son capaces de sentir de forma similar a los humanos. Consideración y respeto atañen a la especie humana, como una obligación prima facie hacia otras especies. Para llegar a tal conclusión no hace falta acudir al principio de «igualitarisno biocéntrico» ${ }^{54}$, sino a los datos que demuestran la influencia de las acciones en el medio ambiente, en las generaciones futuras, en el bienestar de las especies, etc. Esta manera de entender la «igual consideración de intereses» llevará al reconocimiento de derechos para los no humanos, pero no en el sentido en que hablamos de derechos fundamentales o de Derechos Humanos.

\section{DERECHOS Y DEBERES}

En gran medida, el principio de igual consideración de intereses dependía del argumento de continuidad entre las especies. 
Ahora bien, existen por lo menos dos formas de entenderla. En sentido fuerte, la continuidad propicia la analogía entre «pacientes» y «agentes» morales. Esto significa que unos $y$ otros son realmente merecedores de un trato similar, que no sea cruel ni dañino. Las versiones biocéntricas suelen hacer uso de esta forma del argumento. Aquí el tema estará en si la continuidad fuerte lleva a una restricción bien definida de la conducta humana. Por el contrario, la continuidad en sentido débil hacía hincapié en las diferencias o jerarquías entre humanos y no humanos, o en el desigual valor intrínseco de las especies. El antropocentrismo utiliza esta segunda modalidad, por razones perfectamente comprensibles. No llega a la igual consideración de intereses; a cambio establece algunas obligaciones que ha de asumir la especie humana. En fin, ambas versiones han estado abogando en favor de la consideración moral de los animales no humanos, aunque con estrategias distintas. Dicho de forma resumida: en un caso se pretende el bienestar ${ }^{55}$ de los animales no humanos, en otro se restringe la conducta humana para no causar daños irreparables en las especies no humanas. Por tanto, el primer paso para cambiar la perspectiva tradicional ha sido dado, a través de la nueva valoración de intereses y de capacidades. Más complicado resulta el paso siguiente, el asumir que los animales tienen «derechos», en el sentido usual del término. De nuevo, habrá para esto dos respuestas, una «fuerte» y otra «débil».

1. 9 ) La continuidad en sentido fuerte diluye las fronteras ${ }^{56}$ entre especies, minimiza también la asimetría entre humanos y no humanos ${ }^{57}$. Precisamente por eso, requiere luego cierta distancia entre «destinatarios» - los humanos-y «beneficiarios» de los nuevos derechos, no humanos, ya que éstos no están en posición de elevar una demanda. De forma similar a como se hablaba de «agentes» y de «pacientes» morales. No era imprescindible que éstos fueran agentes reflexivos, y para universalizar derechos es suficiente que tengan sensibilidad, autonomía ${ }^{58}$. Ahora bien, continuidad en su sentido fuerte difumina en parte la conexión derechos-deberes, al dejar abiertas las opciones sobre cómo restringir de manera eficaz la conducta de los humanos, auténticos destinatarios de los derechos en beneficio de otras especies. En un principio, estos derechos nunca son presentados como algo dado, ya que no existen de antemano. Son más bien convenciones ${ }^{59}$, «permisos» que dependen en realidad de la legislación vigente, del legislador, del contexto, etc. Si los «derechos positivos» dependen de instituciones o de convenciones, los «derechos morales» tienen validez como pretensiones ${ }^{60}$. Las pretensiones racionales indican lo que se debería hacer. Su validez no dependerá de que sea o no sean realizados, ni de que existan deberes correlativos. En este sentido, los derechos de los animales o bien se han de entender como «derechos morales» -en tal caso había que preguntar por las instituciones que podrían garantizarloso bien se entienden como derechos por analogía, de modo similar a como hablamos de «derechos humanos». De ser así, el prejuicio de especie volvería a aparecer $\mathrm{y}$, ante todo, los derechos podrían ser ampliados de forma ilimitada, en defensa de todos los eventuales beneficiarios, los seres vivos, las plantas ${ }^{61}$, incluso los artefactos, el «medio ambiente» 62 artificial...

2.9) La continuidad en sentido débil subraya que el valor intrínseco es gradual ${ }^{63}$. Por lo mismo, llama la atención sobre la dificultad de establecer una relación contractual con animales no humanos. En cambio, esta versión da lugar a todo tipo de analogías, que favorecen la institucionalización del respeto hacia los animales, los necesarios cambios en la legislación para proteger a otras especies, la sanción a quienes actúan de forma cruel, etc. En este caso se tratará de derechos $\sin$ reciprocidad ${ }^{64}$, sin deberes corre- 
lativos, derechos entendidos como pretensiones, demandas... de los humanos. Por lo tanto, la especie humana confiere derechos negativos ${ }^{65}$ en favor de los animales no humanos. También se puede hablar de derechos «en relación a» 0 «con respecto a» los animales, concernientes a los animales $^{66}$, etc. En definitiva, sirven para limitar el comportamiento de los humanos, más que para proteger directamente un interés ${ }^{67}$, el de los animales no humanos. Desde este enfoque antropocéntrico, los derechos de los animales son siempre una responsabilidad ${ }^{68}$ que recaerá invariablemente sobre la especie humana, como agentes racionales y competentes. Por todo ello, parece obvio que estos derechos se refieren en realidad a los «deberes» 69 de la especie humana. Los «derechos» 70 hacen pensar en obligaciones correlativas, en cambio, éstas son algo que se ha de hacer, sin necesidad o con independencia de que existan derechos correlativos. De un lado, están las obligaciones que corresponden a una demanda o un derecho. De otro, están también aquellas obligaciones de tipo general, que no derivan de una demanda o una pretensión anterior. En este segundo sentido hablamos de «deber moral» ${ }^{71}$. Por lo tanto, cabe referirse de las dos maneras a los deberes hacia los animales. Incluso se puede hablar de «obligaciones indirectas», en la terminología de la Filosofía moderna ${ }^{72}$, con un uso nuevo, a favor de los animales no humanos y del medio ambiente

\subsection{Contra la crueldad}

A pesar de no haber asumido la tesis radicales sobre la «comunidad biótica» - ya resulta discutible la idea de una «comunidad» humana bien integrada-, el antropocentrismo moderado incorpora de alguna manera la consideración moral de los no humanos. Por tanto, rechazará las prácticas crueles, la experimentación con ani- males ${ }^{73}$ que no sea estrictamente necesaria o que provoque sufrimientos innecesarios; rechazará asimismo ciertas formas de caza y de ganadería que atentan gravemente contra el bienestar de los no humanos, y que provocan un sufrimiento inútil. Está en juego no sólo el bienestar de otras especies sino, al mimo tiempo, la calidad de vida ${ }^{74}$ de la propia especie. Veremos luego el tema, complicado, de cómo restringir de manera eficaz y cómo modificar las conductas de los «agentes» morales. Ahora se revisarán las razones en contra del sufrimiento de los «pacientes» morales. Así, por ejemplo, el modelo kantiano ${ }^{75}$ concede siempre primacía a la especie humana, no obstante el bienestar de los animales formará parte de las obligaciones prima facie, obligaciones que conciernen a los humanos. Por lo general, el biocentrismo ha puesto en duda la fuerza de tales argumentos centrados en los humanos, ya que éstos dejan las prioridades tal y como estaban: ¿cómo persuadir a la especie humana de las eventuales ventajas del altruismo interespecífico? De hecho, no se prodigan demasiado las actitudes de benevolencia entre humanos y menos aún la benevolencia hacia los no humanos. La cuestión admite dos interpretaciones, una de corte biocéntrico y otra de corte antropocéntrico: el principio del daño, el principio de preservación.

a) El principio del daño fue introducido por J. S. Mill ${ }^{76}$ para fijar los límites del poder de la sociedad sobre los individuos. Evitar el daño a los demás sería la única razón para interferir en las acciones individuales. El biocentrismo usa el criterio del daño para limitar las conductas humanas y para proteger a los seres vivos, el medio ambiente en su conjunto. Por las mismas razones que defiende la continuidad entre las especies, el valor intrínseco de cada una de éstas, los «derechos de los animales». Sin embargo, se trata aquí de un uso derivado, o por analogía, ya que el «daño» se aplicaba a las relaciones entre 
individuos de la especie humana, y en el contexto de sociedades liberales. Es más, incluso en su forma más abierta, como principio «prima facie», el daño no resolvería de forma satisfactoria los frecuentes conflictos de intereses entre animales no humanos y animales humanos. Valga como ejemplo el dilema referido por A. Naess ${ }^{77}$ : ¿se debía proteger a los lobos que amenazaban a los habitantes de una zona de Noruega? ¿Había que contratar cazadores para los lobos o «pastores» para los niños? En España, y a propósito del Plan Hidrológico Nacional, se discutió en el 2000 sobre el impacto ambiental de los nuevos embalses sobre algunas especies, como las poblaciones de mustélidos ${ }^{78}$. ¿Cómo entender aquí la igual consideración de intereses?

b) El principio de preservación se refiere a especies, no a individuos. Pretende la restricción de la conducta humana, en beneficio de otros seres vivos, del medio ambiente, y de los propios humanos. Pues la visión antropocéntrica es perfectamente compatible con el interés - antropocéntrico en su origen- por mantener la variedad de especies. Por lo demás, el valor intrínseco reside en las especies, no en los individuos; debido a ello, éstos serán reemplazables o irreemplazables, según su grado de evolución y su distribución ${ }^{79}$ en los ecosistemas. Por tanto, este principio está cortado a la medida de un tipo de antropocentrismo no radical, que cuestiona los privilegios de especie $\mathrm{y}$, al mismo tiempo, se apoya en la separación entre «agentes» $y$ «pacientes» morales, entre «destinatarios» y «beneficiarios» de la acción. Así se dirá que existen obligaciones -indirectas o «mediadas» ${ }^{80}$ para los humanos, más que «derechos» para los no humanos. En cambio, este enfoque antropocéntrico vincula la supervivencia de las especies $y$, en general, los temas ambientales al bienestar humano. ¿Preservación y democracia? En buena medida, la discusión sobre estas cuestio- nes será tanto más abierta cuanto más lo sea la esfera pública, por lo que la participación democrática favorece la transparencia sobre el uso de los recursos y sobre las políticas ambientales.

No hay un argumento definitivo ${ }^{81}$ para terminar de una vez por todas con el sufrimiento innecesario en los animales no humanos ${ }^{82}$. Existen recomendaciones, argumentos indirectos, compromisos más o menos sólidos, principios que justifican un cambio de actitud, a fin de eliminar ese componente de ensañamiento y de crueldad. Pues tales conductas son incompatibles con una sensibilidad ambiental bien desarrollada. De un lado, el biocentrismo subraya el valor intrínseco de los seres vivos. Tal solución no asegura el siguiente paso, el cambio efectivo en las actuaciones de los humanos. De otro lado, el antropocentrismo recurre a menudo a los argumentos resbaladizos: la crueldad es degradante para quien la practica, no sólo para quien la padece. Para mantener y elevar los estándares de civilización ${ }^{83}$, no se deberá hacer a los animales lo que tampoco se debe hacer a los humanos.

\section{JUSTICIA INTERESPECIFICA}

Ciudades y bosques podrían arder durante días enteros. Ríos envenenados, aire como fuego, seres abrasados, ciegos, enfermos, destrucción indiscriminada. Carcasa cancerosa de nuestros hijos... A. Roy ${ }^{84}$ ha insistido en tales términos sobre el potencial devastador del armamento nuclear. Un futuro amenazado, graves enfermedades para las generaciones venideras, escasez de recursos, todo ello debería ser un argumento lo suficientemente persuasivo como para hacer un uso responsable de la energía y del medio ambiente. Los bosques arderán, pero también lo harán las ciudades. Podemos preguntarnos qué sucedería si todo ello, el daño irreversible para la especie humana, no fuera un motivo váli- 
do para corregir las políticas ambientales. De no ser aceptado, ¿sería posible la consideración y el respeto por los animales no humanos? Digamos que la «justicia entre generaciones» crea las condiciones favorables para construir la «justicia entre especies» ${ }^{85}$. El antropocentrismo moderado ha recurrido a este argumento, el bienestar de las generaciones futuras, con el objetivo de analizar las cuestiones de medioambiental, incluyendo el bienestar de los animales no humanos.

1.9) El antropocentrismo parte de una consideración asimétrica, en la cual «agentes» $y$ «pacientes» morales son, respectivamente, «destinatarios» $y$ «beneficiarios» de la acción. La continuidad de las especies, en su sentido débil, facilitaba la atribución de responsabilidad y de obligaciones a los humanos, como agentes competentes que son. De este modo, se podía hablar con toda razón de «derechos de los animales», pero sólo en sentido derivado o analógico. Se trataba, en realidad, de «deberes indirectos» que concernían más bien a la especie humana. Por eso eran deberes prima facie, como la obligación de no causar daño, o la compadecerse $^{86}$ de los seres vivos que padecen sufrimiento, etc. Los argumentos resbaladizos pretendían dar más consistencia a los motivos que inducen a restringir la conducta humana, o para eliminar la crueldad, aportando así beneficios directos para los humanos, beneficios indirectos para los no humanos. En cualquiera de los casos, el alcance de los riesgos ambientales termina por anular definitivamente las fronteras entre especies. Ríos envenenados, aire como fuego, seres vivos abrasados, ciegos, enfermos...

2.9) El argumento del futuro ensancha las perspectivas de la ética medioambiental. Pues permite analizar todo tipo de relaciones asimétricas, como las que mantienen los humanos con los no humanos, también las relaciones entre las generaciones actuales y las generaciones futuras ${ }^{87}$.
Un tipo de relaciones $\sin$ reciprocidad, como resulta obvio. Los no humanos no cuentan con las competencias de habla y de acción, propias de los humanos. Tales competencias definen a los agentes, a los cuales se refería la Teoría de la justicia de J. Rawls. Teoría que ya contemplaba tanto los problemas básicos de la reciprocidad como ciertas obligaciones hacia las generaciones futuras ${ }^{88}$ : ¿las posibles ventajas compensan los sacrificios que habría que hacer en la actualidad? Algunas teorías de la justicia ${ }^{89}$ han introducido este concepto de «justicia entre generaciones», o «justicia transgeneracional» ${ }^{90}$, como condición para llegar a la «justicia entre especies» ${ }^{91}$. Lo cual demuestra indirectamente que los principios biocéntricos no son imprescindibles cuando se desea analizar las cuestiones ambientales ${ }^{92}$. La perspectiva de generaciones como depositarias, solidarias o «guardianas» ${ }^{93}$ del bienestar de otras generaciones favorece, en general, una actitud más responsable hacia el medio. En beneficio de los humanos y de los no humanos.

3.9) La consideración de los intereses de las generaciones futuras justifica indirectamente la preservación de los recursos y de la variedad de especies. Esto es, justifica un uso restringido de tales recursos. Afirmar que las actuales generaciones tienen sólo la custodia del medio ambiente, implica entonces que éstas sí tienen obligaciones hacia las generaciones venideras. Las tienen hoy ${ }^{94}$, aunque no sepan, ni puedan saber si esas generaciones llegarán a existir alguna vez en el futuro. Tal responsabilidad se refiere a las generaciones de humanos $\mathrm{e}$, indirectamente, a las generaciones de no humanos. Está claro, de otra parte, que las obligaciones hacia personas potenciales ${ }^{95}$ no serán exactamente como aquellas que se tienen hacia personas reales: por evidentes problemas de información, debido a su contingencia e indeterminación ${ }^{96}$. Lo cual no significa que no se tenga ninguna clase de obligación hacia 
esas personas potenciales. Dentro de todas las limitaciones mencionadas, podemos hablar entonces de «deberes imperfectos» $\mathrm{y}$ de «deberes indirectos» hacia quienes verán las consecuencias de decisiones que se están tomando hoy. O tal vez no lleguen a verlas, a causa de decisiones erróneas, por estar creando un entorno hostil para los seres vivos. En cualquiera de los casos, la perspectiva de generaciones y de especies en lucha, compitiendo entre sí, habría de dar paso a otra versión bien diferente, en la que existiera una suerte de cooperación ${ }^{97}$ entre generaciones y especies diversas. No a pesar, sino precisamente porque son desiguales.

En suma, la expansión de lo moral y de la Ética medioambiental debe mucho a la creciente sensibilidad hacia las necesidades de otras especies. También es deudora de una información fiable sobre los efectos desencadenados en el medio ambiente y en la supervivencia de las especies por una conducta humana irrestricta. El conocimiento de otras especies y de su conducta induce a un cambio en las actitudes hacia los animales y hacia el medio ambiente. Sin embargo, esta nueva expansión de la moralidad tiene un límite bien definido, ya que nunca podrán situarse los humanos en el lugar de los no humanos, a fin de respetar sus intereses tal y como realmente son. Ya es complicado hacerse cargo de las necesidades que tienen los individuos de la propia especie humana... Por lo tanto, se puede esperar una conducta más sensible y más civilizada, que sea resultado de una conciencia ambiental desarrollada ${ }^{98}$. Pero este paso, aun siendo fundamental, no asegurará una consideración moral plena, dada la asimetría entre especies. Las emociones ${ }^{99}$, las actitudes favorables hacia los intereses de los animales no humanos son meritorias, señal inequívoca de buena voluntad y de un altruismo que emerge más allá de las fronteras convencionales entre las especies. Ahora bien, estos cambios de actitud entran, en gran medida, en el ámbito de lo que se suele denominar lo «supererogatorio» ${ }^{100}$. Esto es, aquello que es meritorio, beneficioso para otros, pero no es exigible. No es una obligación. Al menos, no una obligación directa. Por eso mismo, tiene todavía sentido el lenguaje de la justicia y de los derechos en el campo de la Ética medioambiental. Para asegurar compromisos duraderos con el bienestar de humanos y de no humanos. Los «derechos» de los animales definen, pues, las obligaciones de la especie, de forma relativamente estable e independiente de las actitudes particulares de los individuos hacia las demás especies.
1 A. Roy, «The Greater Common Good», The Cost of Living, Londres, Flamingo, 1999, pp. 5-114.

${ }^{2}$ Sobre la confluencia de lo ambiental y lo político, A. Lipietz, «Ecología política y movimiento obrero: similitudes y diferencias», Revista de Occidente, 167, 195, pp. 95-107. K. Schrader-Frechette recuerda que, por lo general, los temas ambientales se han orientado en dos direcciones, hacia lo científico o, por el contrario, hacia lo político, «Practical Ecology and Foundations for Environmental Ethics», The Journal of Philosophy, núm. 92, 1995, pp. 621-635. P. Christoff se refiere incluso a una forma de democracia que esté «ecológicamente guiada», «Ecological Citizens and Ecologically Guided Democracy», en B. Doherty, M. Degeus, Democracy and Green Political Thought, Londres, Routledge, 1996, pp. 151-169. R. E. Goodin ha defendido claramente la necesidad de integrar los programas políticos junto a las demandas de carácter ambiental, «What's New», Green Political Theory, Cambridge, Polity Press, 1992, pp. 1-18. H. J. McCloskey ha señalado, en este mismo sentido, las diferencias radicales entre sociedades democráticas, abiertas, y sociedades que se encuentran bajo una organización dictatorial. Los dos tipos de sociedad reaccionan de forma diferente ante el debate y las reformas, acordes con las nuevas demandas ambientales, «The Politics 
of Ecological Reforms: The Liberal Democratic Social Order», en Ecological Ethics and Politics, Totowa, Rowman, 1983, pp. 156-159. Por su parte A. Valencia Saíz ha analizado el ecologismo como ideología política, «Teoría política verde: balance de una disciplina emergente», en Revista Española de Ciencia Política, 3, 2000, pp. 181-194.

3 Sobre el antropocentrismo en cuestiones ambientales M. T. López de la Vieja, «Justicia en la Ética medioambiental», Principios morales y casos prácticos, Madrid, Tecnos, 2000, pp. 176-233.

4 J. Mansbridge, S. Moller Okin, «Feminism», en R. E. Goodin, Ph. Pettit, A Companion to Contempo rary Political Philosophy, Cambridge, Blackwell, 1993, pp. 269-290.

s Ch. Taylor, «The Politics of Recognition», Ch. Taylor, Multiculturalism and «The Politics of Recognition», Princeton, Princeton University Press, 1992, pp. 25-73.

6 R. Dworkin, «Rights as Trumps», en J. Waldron, Theories of Rights, Oxford, Oxford University Press, 1992, pp. 153-167.

7 Según la conocida expresión de C. Gilligan, «In a Different Voice: Women's Conceptions of Self and of Morality», en Harvard Educational Review, núm. 17, 1977, pp. 481-517.

$8 \mathrm{~T}$. Benton menciona expresamente los avances impulsados por las Teorías feministas y, en contraposición, las dificultades con que tropiezan casi siempre las teorías que se interesan más bien por los derechos de los no humanos, «Introduction», Natural Relations, Londres, Verso, 1993, pp. 1-19. En la primera edición de su obra sobre la emancipación de los animales, $P$. Singer se refirió ya a los movimientos de liberación de la mujer, para rechazar que éstos hayan sido la última frontera contra los prejuicios y la opresión, «Preface to the 1975 Edition», en Animal Liberation, Londres, Pimlico, 1995, pp. IX-XV. Cabe añadir tan sólo que este tipo de comentarios suelen mostrarse poco sensibles a la situación en que se encuentra, de hecho, la «igual consideración» de las mujeres.

9 Una obra tan amplia y tan compleja como la de R. Brandom mantiene aún fuertes diferencias, de tipo cualitativo, entre seres humanos — capacidad para sentir, para saber, capacidad discursiva - y mamíferos superiores, con capacidad para sentir, en Making It Explicit, Cambridge, Harvard University Press, 1994, p. 276.

10 P. Singer, «Prologue», en In Defence of Animals, Oxford, Blackwell, 1986, pp. 1-10.

11 «On Comparing the Value of Human and Nonhuman Life», en E. Morscher, O. Neumaier y P. Simons, Applied Ethics in a Troubled World, pp. 93-104.

${ }_{12}$ T. Regan, «Ethical Thinking and Theory», en The Case for Animal Rights, Berkeley, University of California Press, 1985, pp. 121-149; «The Case for Animal Rights», en P. Singer, In Defence of Animals, pp. 13-26.
${ }_{13}$ T. Regan, «Animal Rights, Human Wrongs», en H. B. Miller y W. Williams, Ethics and Animals, Clifton, Humana Press, 1983, pp. 19-43. B. E. Rollin también insistía sobre este argumento, que la racionalidad no ha de ser relevante para aceptar la consideración moral, «The Legal and Moral Bases of Animal Rights», en H. B. Miller y W. Williams, Ethics and Animals, pp. 103-118.

14 «Rights Across Species», en E. Morscher, O. Neumaier y P. Simons, Applied Ethics in a Troubled World Dordrecht, Kluwer, 1998, pp. 41-55

15 H. B. Miller presentaba una tipología para ordenar estas teorías, interesadas en definir de modo convincente el status moral de los animales, «Intoduction. "Platonist" and "Aristotelians"», en H. B. Miller, y W. Williams, Ethics and Animals, pp. 1-14

16 P. Singer rechazó esta versión, que confunde la afectividad hacia los animales con algo diferente, los principios de una moralidad mucho más amplia, «Preface to the 1975 Edition», en Animal Liberation, pp. IX-XV.

17 F. B. de Waal critica la «humanización» de los animales. Este enfoque ha motivado que incluso la ciencia busque en los animales cualidades equivalentes a las de los humanos, «Anthropomorphism and Anthropodenial: Consistency in Our Thinking About Humans and Other Animals», 27, 1999, pp. 255-280.

18 D. R. Griffin, «Nonhuman Minds», en Philosophical Topics, núm. 27, 1999, pp. 233-254.

19 Sobre el tema de la protección de los animales, B. Irrgang, «Tierschutz», W. Korff, L. Beck, P. Mikat, Lexikon der Bioethik, Gütersloh, Gütersloher Verlagshaus, 1998, pp. 561-567.

20 P. Singer, «All Animals Are Equal...», en Animal Liberation, pp. 1-23.

${ }^{21}$ B. E. Rollin, en el artículo citado antes, «The Legal and Moral Bases of Animal Rights», en H. B. Miller y W. Williams, Ethics and Animals, pp. $103-118$

22 J. Rachels, «Do have Animals a Right to Life?», en H. B. Miller y W. Williams, Ethics and Animals, pp. 275-284.

23 R. G. Frey, «On Why We Would Do Better to Jettison Moral Rights», en H. B. Miller y W. Wiliams, Ethics and Animals, pp. 285-301.

${ }^{24}$ R. Dworkin, «What Rights Do We Have?», Taking Rights Seriously, Cambridge, Harvard University Press, 1977, pp. 266-278.

${ }_{25} \mathrm{P}$. Westen señalaba que «igualdad» puede ser tanto un término descriptivo como un término de carácter evaluativo, «The Persuasiveness of Equality», en Speaking of Equality, Princeton, Princeton University Press, 1990, pp.

${ }_{26}$ D. Mayo, «Against a Scientific Justificaction of Animal Experiments», en H. B. Miller y W. Williams, Ethics and Animals, pp. 339-359.

27 Sobre el problema de las condiciones de vida en las granjas, el estudio de W.B. Gross «Chicken-Envi- 
ronment Interactions», en H. B. Miller y W. Williams, Ethics and Animals, pp. 329-337. Una crítica de las técnicas aplicadas en las granjas se encontraba en el artículo de J. Mason, «Brave New Farm?», en P. Singer, In Defence of Animals, pp. 89-107.

${ }_{28}$ Este tema, la caza como una práctica a evitar prima facie, ha sido tratado por P. S. Wenz, «Ecology, Morality, and Hunting», en H. B. Miller y W. Williams, Ethics and Animals, pp. 183-197.

29 R. Attfield analizaba las bases para la consideración moral de los no humanos, como tener intereses, preferencias, capacidad para ser dañado o recibir los beneficios de la actuación de otros, etc. «Preferences, Health, Interest, and Value», en The Electronic Journal of Analytic Phylosophy, núm. 3, primavera 1995. Este mismo autor se ha referido a los criterios de 1 consideración moral, como seres humanos, seres posibles, del futuro, del pasado, seres no humanos, «EI ámbito de la moralidad», en J. M. García Gómez Heras, Ética del medio ambiente, Madrid, Tecnos, 1997, pp. 71-88.

${ }^{30}$ Sobre la debilidad del prejuicio de especie, H. La Follette y N. Shanks, «The Origins of Speciecism", en Philosophy, núm. 71, 1996, pp. 41-61. Contra el «chauvinismo» de los humanos, R. Routley y V. Routley, "Against the Inevitability of Human Chauvinism», en R. Elliot, Environmental Ethics, Oxford, Oxford University Press, 1995, pp. 104-128.

"P. Singer, «Expanding the Circle of Ethics», The Expanding Circle, Oxford, Oxford University Press, 1983, pp. 111-124.

32 J. M. Buchanan, «Moral Community and Moral Order», en H. B. Miller y Williams, Ethics and Animals, pp. 95-102

${ }^{33}$ P. W. Taylor, «In Defense of Biocentrism», en Environmental Ethics, núm. 5, 1983, pp. 237-243.

${ }^{34}$ A. Naess, «The Shallow and the Deep, LongRange Ecology Movement. A Summary», en Inquiry, núm. 16, 1973, pp. 95-100. J. Ferrater Mora recogía otra expresión similar, «igualdad viviente», al referirse a P. Singer, Diccionario de Filosofia, Madrid, Alianza, 1980, vol. A-D, pp. 161-163.

${ }^{35}$ M. W. Fox, «Philosophy, Ecology, Animal Welfare, and the «Rights» Question», en H. B. Miller, W. Williams, Ethics and Animals, pp. 307-315.

${ }^{36}$ K. E. Goodpaster repasaba los criterios para atribuir a los seres vivos relevancia moral, «On Being Morally Considerable», The Journal of Philosophy 1978, pp. 308-325

37 A. Naess unía ambos objetivos, el reconocimiento del valor intrínseco de los animales y, de otro lado, la necesidad de combatir actividades destructivas, "A Defence of the Deep Ecology Movement», en Environmental Ethics, núm. 6, 1984, pp. 265-270.

$38 \mathrm{~T}$. Regan criticaba abiertamente este enfoque, el «uso» del medio ambiente, «The Nature and Possibility of an Environmental Ethics», en Environmental Ethics, núm. 3, 1981, pp. 19-34. En varias de sus obras, H. Rolston III ha rechazado también el enfoque instrumental sobre la Naturaleza, «Value in Nature and the Nature of Values,, en Philosophy, Supplement 36, Cambridge, Cambridge University Press, 1994, pp. 13-30; «The Concept of Natural Value: A Theory for Environmental Ethics», en Environmental Ethics, Philadelphia, Temple University Press, 1988. pp. 192-245.

39 Sobre la posibilidad de incluir a los primates en la clase de las personas, A. Gomila Benajem, «Personas primates», en J. M. García Gómez-Heras, Ética del medio ambiente, pp. 191-204

40 J. B. Callicot, «Elements of an Environmental Ethic: Moral Considerability and the Biotic Community», en Environmental Ethics, núm. 1, 1979 pp. 71-81.

41 P. W. Taylor, en el artículo ya citado, «In Defense of Biocentrism», en Environmental Ethics, núm. 5, 1983, pp. 237-243.

42 De ahí las criticas más fuertes hacia la «moral humanista», ya que ésta limita la compasión y la solidaridad al propio grupo humano, como ha explicado J. Mosterin, «Consideración moral de los animales», en Los derechos de los animales, Madrid, Debate, 1995, pp. 15-30. Este autor se ha referido también abiertamente a la «bancarrota del antropocentrismo», «Niveles de la conciencia moral», en iVivan los animales!, Madrid, Debate, 1998, pp. 195-209.

43 Por el contrario el biocentrismo sí establece claramente la diferencia entre «antropocéntrico» y «antropogénico», tal como hacía H. Rolston III en su conocido artículo «Value in Nature and the Nature of Value», en Philosophy, Supplement 36, Cambridge, Cambridge University Press, 1994, pp. 13-30

44 J. Habermas, «The European Nation State. Its Achievements and Its Limitations. On the Past and Future of Soveregnity and Citizenship», en Ratio Juris, núm. 9, 1996, pp. 125-137.

45 «... and finally, we may ask whether justice can be extended to our relations to animals and the order of nature. As I have said (I: 3.4 ), I believe that justice as fairness can be reasonably extended to cover the first three problems...», J. Rawls, en Political Liberalism, Nueva York, Columbia University Press, 1993 p. 245 .

$46 \mathrm{M}$. Warnock no ve injusticia alguna en las actitudes de "especieísmo», ya que los humanos son, en realidad, los únicos beneficiarios de los principios morales, «Man and Other Animals», en The Uses of Philosophy, Oxford, Blackwell, 1992, pp. 9-23.

47 J. Sterba defiende el principio de defensa y el principio de preservación humana, a pesar de estar en desacuerdo con la postura antropocéntrica clásica. Considera que las diferencias entre especies no permiten deducir que exista alguna clase de superioridad de unas sobre otras, «From Anthropocentrism to Nonanthropocentrism», en Justice for Here and Now, Cambridge, Cambridge University Press, 1998, pp. 125-150. 
48 E. Tugendhat, «Wer sind alle?», en A. Krebs, Naturethik, Frankfurt, Suhrkamp, 1997, pp. 1000-110.

49 F. Savater comentaba la dificultad de razonar sobre el valor intrínseco de lo natural, Las preguntas de la vida, Barcelona, Ariel, 1999, p. 181. Este auto criticaba asimismo el concepto de daño o de sufrimiento infringido a la Naturaleza, «Madre dolorosa», El País, 2 de octubre, 1995, p. 13.

50 T. Regan utiliza también esta terminología para analizar el tema de las diferencias entre quienes pue den justificar moralmente sus acciones y quienes, en cambio, no están en condiciones de hacerlo así, «Indirect Duty Views», en The Case for Animal Rights, pp. 150-194.

s1 R. M. Hare se servía precisamente del argumento del interés de las personas para apoyar el principio de conservación, «Moral Reasoning About the Environment», en B. Almond, D. Hill, Applied Philosophy, Londres, Routledge, 1991, pp. 9-20

52 Para el elemento relacional en la noción de daño, P. de Lora, «La vida como mal», en Claves de la razón práctica, núm. 113, junio de 2001 , pp. 45-53.

$53 \mathrm{~T}$. Regan se mostraba partidario de un «vegetarianismo condicionado». Es decir, una práctica vegetariana que es coherente con la voluntad de no causar daño y que, a la vez, permite el consumo de carne en determinadas situaciones, «The Moral Basis of Vegetarianism», en Canadian Journal of Philosophy, 1975, pp. 181-214. Para este mismo tema, M. Tafalla, «Seis buenas razones para ser vegetariano», en M. Tafalla (ed.), Los derechos de los animales, Barcelona, Idea Books, 2003, pp. 171-175.

:54 R. Attfield defiende el reconocimiento de obligaciones hacia el medio, hacia los animales, hacia las generaciones futuras. Llega a esta conclusión sin necesidad de asumir una versión estricta de «igualitarismo biológico», «The Comprehensive Ecology Movement», en E. Morscher, O. Neumaier, y P. Simons, Applied Ethics in a Troubled World, pp. 9-27.

$55 \mathrm{~S}$. Castiglione considera que el bienestar y los derechos de los animales son un tema con entidad propia, dentro de las cuestiones que se refieren de forma general al medio ambiente, «Introduzione», en I diritti degli animali, Bologna, Il Mulino, 1988, pp. 9-36.

s6 Un aspecto interesante de la continuidad, o de la desaparición de fronteras entre animales, humanos y máquinas era tratado desde un punto de vista feminista en el trabajo de D. Haraway, Ciencia, «cyborgs» $y$ mujeres, Madrid, Cátedra, 1991, pp. 251-311.

57 A este respecto son relevantes los datos que ya se tienen sobre las capacidades de algunas especies, «D. M. Rumbaugh, S. Savage-Rumbaugh, «Apes and Language Research», en H. B. Miller y W. Williams, Ethics and Animals, pp. 207-217.

$58 \mathrm{~S}$. Castiglione propone la autonomía, la sensibilidad, tener caracteres vitalmente importantes, etc., como atributos de los agentes morales, «The Problem of Animal Rights», en ARSP, Beiheft, 58, 1995, pp. 210-214.

s9 J. Mosterín ha defendido este carácter convencional, «Derechos humanos, derechos de los animales», Los derechos de los animales, pp. 31-45; «Derechos de los animales», en iVivan los animales!, pp. 311-325; «Creando derechos», en El País, 29 de agosto, 1999, p. 12.

${ }_{60}$ R. Harre y D. L. Robinson recogen la definición de derechos como pretensiones, «On the Primacy of Duties», en Philosophy, núm. 70, 1995, pp. 513-532. Por su parte, J. Feinberg analizaba los derechos positivos y los derechos morales, considerados como pretensiones, racionalmente demostrables, «Rights», en W. E. Reich, Encyclopedia of Bioethics, Londres, The Free Press, 1978, pp. 1507-1511.

61 Derechos de los árboles? A. De-Shaalit planteaba esta cuestión, «Introduction», en Why Posterity Matters, Londres, Routledge, 1998, pp. 1-12.

62 Un aspecto menos tratado de la conservación ambiental, del que se ocupaba F. Broncano, «Los viejos cacharros nunca mueren. Un argumento ético a favor de la conservación del medio ambiente artificial», en J. M. García Gómez-Heras, Ética del medio ambiente, pp. 179-190. Sobre este tema de la conservación de seres no vivos, D. Jamieson, «The City Around Us», en T. Regan, Earthbound, Philadelphia, Temple University Press, 1984, pp. 38-73.

63 Sobre el valor gradual de las especias, J. Wetlesen, «Animal Rights or Human Duties?», en ARSP, Beiheft, 61, 1995, pp. 163-170

64 F. Savater argumentaba sobre la falta de reciprocidad en « ¿Todas sus criaturas?», en El País, 29 de agosto, 1999, p. 12.

$65 \mathrm{M}$. Warnock se muestra partidaria de esta versión, los derechos conferidos por los humanos. Derechos entendidos como "deberes» que éstos tienen hacia los no humanos, «Rights», en An Intelligent Person's Guide to Ethics, Londres, Duckworth, 1998, pp. 54-74.

66 J. Feinberg analiza esta solución, derechos «hacia» 0 «concernientes a» los animales, «The Rights of Animals and Unborn Generations", en Rights, Justice , and the Bounds of Liberty, Princeton, Princeton University Press, 1980, pp. 159-184.

67 Los derechos como protección de un interés eran analizados por J. Waldron, «Rights», en R. Goodin y $\mathrm{Ph}$. Pettit, A Companion to Contemporary Political Philosophy, Oxford, Blackwell, 1993, pp. 575-585.

$68 \mathrm{M}$. Van Hoecke presenta los derechos como nuevas formulaciones de los deberes de la humanidad, «Confusion on the Concept of "Rights": The Case of the So-Called "Animal Rights"), en ARSP, Beiheft, 58, 1995, pp. 215-225

${ }^{69}$ M. T. López de la Vieja, Principios morales y casos prácticos, pp. 206-207.

70 A. White, «The Variety of Rights», en Rights, Oxford, Clarendon, 1984, pp. 13-19. 
7 J. Feinberg recordaba el uso general del término «deber», llegando a la conclusión de que existe un deber que concierne a la especie humana, preservar todas las especies, no privar a los propios descendientes de algo valioso, etc., «Human Duties and Animal Rights», en Rights, Justice, and the Bounds of Liberty, pp. 185-206.

${ }_{72}$ I. Kant, «Vorlesungen über Moralphilosophie», en Gesammelte Schriften, Berlin, De Gruyter, 1974, vol. XXVII, pp. 458-463.

${ }^{73}$ R. D. Ryder hizo una revisión de los argumentos a favor y también de las alternativas a la experimentación con animales, «Speciesism in the Laboratory», en P. Singer, In Defence of Animals, pp. 77-88.

${ }^{74}$ Autores como H. Spira asocian la liberación de los animales con la «liberación de los humanos», en el sentido de que el bienestar de los animales afecta a la calidad de vida de los humanos, «Fighting for Animal Rights», en H. B. Miller y W. Williams, Ethics and Animals, pp. 373-380.

75 M. G. Hansson comentaba el enfoque kantiano y los deberes prima facie de la especie humana hacia los animales, «Kantian Ethics Applied in the Area of Biomedicine», en Human Dignity and Animals Wellbeing, Uppsala, Almqvista. Wiksell, 1991, pp. 157 198.

76 J. S. Mill, On Liberty, Londres, Penguin, 1985, p.68.

77 A. Naess en el artículo citado antes, «A Defence of the Deep Ecology Movement», en Environmental Ethics, núm. 6, 1984, pp. 265-270. Recogí las tesis centrales del autor y comenté este caso en Principios morales y casos prácticos, pp. 196-197.

78 «La nutria, otra víctima del Plan Hidrológico», en El Mundo, 30 de octubre de 2000, p. 40.

79 G. Patzig se refiere a la jerarquía de las relaciones con lo natural, así como a la jerarquía de las distintas obligaciones, «Ética ecológica dentro de los límites de la mera razón», en Hechos, normas, proposiciones, Barcelona, Alfa, 1986 pp. 201-223.

${ }^{80}$ H. Shue, «Mediating Duties», en Ethics, núm. 98, 1988, pp. 687-704.

81 Para los distintos enfoques sobre el tema M. Tafalla, «Introducción: un mapa del debate», en M. Tafalla (ed.), Los derechos de los animales, pp. 15-39.

${ }_{82}$ P. de Lora ha mostrado las limitaciones del argumento kantiano sobre las obligaciones indirectas hacia los animales, «La Ética de la reciprocidad», en Justicia para los animales, Madrid, Alianza, 2003 pp. 109-134.

$83 \mathrm{~J}$. Habermas introduce el argumento de los deberes hacia los animales como elemento de una sociedad planetaria civilizada, «Erläuterungen zur Diskursethik», en Erläuterungen zur Diskursethik, Frankfurt, Suhrkamp, 1991, pp. 223-226.
${ }^{34}$ A. Roy, «The End of Imagination», en The Cost of Living, pp. 117-161.

${ }^{85}$ D. Van De Veer se refiere a la «justicia interespecífica», como principio para regular las relaciones entre especies, «Interspecif Justice and Animal Slaughter», en H. B. Miller y W. Williams, Ethics and Animals, pp. 147-162.

${ }^{86} \mathrm{U}$. Wolf se refiere a principios prima facie y a la compasión universal, «Haben wir moralische Verflichtungen gegen Tiere?», en A. Krebs, Naturethik, pp. 47-75.

${ }^{87}$ Para el tratamiento deficiente de la temporalidad en las versiones contractualistas, la responsabilidad de unas generaciones para con otras, la transmisión y las obligaciones hacia el futuro, F. Ost, M. Van Hoecke, «Del contrato a la transmisión. Sobre la responsabilidad hacia las generaciones futuras», en Doxa, núm. 22, 1986, pp. $607-630$.

88 J. Rawls, «The Problem of Justice Between Generations», en $A$ Theory of Justice, Oxford, Clarendon, 1972, pp. 284-293.

89 G. Patzig recoge la tesis de que existe la obligación de legar un mundo no devastado, «ecológica dentro de los límites de la mera razón», en Hechos, normas, proposiciones, pp. 219-220

90 A. De-Shalit, «The Transgenerational Community", Why Posterity Matters, Londres, Routledge 1995, pp. 13-50

91 M. T. López de la Vieja, «Justicia entre especies y entre ciudadanos», en J. M. García Gómez-Heras y C. Velayos, Tomarse en serio la naturaleza, Madrid, Biblioteca Nueva, 2004, pp. 43-65.

${ }_{92}$ Por su parte, J. Narveson sostiene que el Contractualismo no es una teoría adecuada para hacer un análisis de este tema, los derechos de los animales, «Animal Rights Revisited», en H. B. Miller y W. Williams, Ethics and Animals, pp. 45-59.

${ }_{93} \mathrm{Ph}$. Van Parijs, «The Disfranchisement of the Elderly, and Other Atemps to Secure Intergenerational Justice», en Philosophy \& Public Affairs, núm. 27,1999 , pp. 292-333

94 Sobre los derechos que son válidos ahora, siendo las generaciones futuras los beneficiarios de tales derechos, G. Pontara, «Teoría de los derechos y generaciones futuras», en Ética y generaciones futuras, Barcelona, Ariel, 1996, pp. 104-132.

$95 \mathrm{M}$. Warren analizaba el tema de los derechos de las personas potenciales, «Do Potential People Have Moral Rights?», en R. Sikora, B. Barry, Obligations to Future Generations, Philadelphia, Temple University Press, 1978, pp. 14-30.

96 A. Baier, «For the Sake of Future Generations», en T. Regan, Earthbound, Philadelphia, Temple University Press, 1984, pp. 214-246.

$97 \mathrm{~T}$. Benton desarrolla la idea de las especies como agentes con capacidad para cooperar, como «partners», en Natural Relations, p. 17. 
.98 Un ejemplo de cómo la nueva sensibilidad ha terminado por incidir en conceptos, que son clave para la Filosofía moral y política, se encuentra en el articulo de C. Velayos, «¿Es posible una ciudadanía ecológica?», en J. M. García Gómez-Heras y C. Velayos, Tomarse en serio la naturaleza, pp. 145-164.

${ }_{99}$ M. P. Golding recogía el papel a desempeñar por los afectos en el tratamiento de las cuestiones ambien- tales, «Future Generations, Obligations To», en W. E. Reich, Encyclopedia of Bioethics, pp. 507-512.

100 J. Feinberg, «The Moral and the Legal Responsability of the Bad Samaritan», en Freedom and Fulfillment, Princeton, Princeton University Press, 1992 pp. 174-196; J. C. Bayon, «Los deberes positivos generales y la determinación de sus límites», en Doxa, núm. 22, 1986, pp. 35-54. 